\title{
Stakeholder Identification as an Issue in the Improvement of Software Requirements Quality
}

\author{
Carla Pacheco and Edmundo Tovar \\ Languages and Informatics Systems and Software Engineering Department \\ Faculty of Computer Science, Polytechnic University of Madrid. Spain \\ cpacheco@zipi.fi.ump.es, etovar@fi.upm.es
}

\begin{abstract}
Stakeholder identification together with its needs and expectations has been poorly realized in software projects. This is probably because the process is mistakenly viewed as a self-evident task in which direct users and the development team are the only stakeholders. It could also be due to the fact that the identification area can be substituted by opinions or knowledge from other more accessible sources of information. This paper provides a review of stakeholder identification literature and an overview of the state-of-the-art in methods for that purpose, which leads to a number of issues that are important in further research (e.g. developing a methodology). The paper findings are presented from two points of view: firstly, the impact of stakeholder identification on software requirements quality, and secondly, practices developed to carry out this task. Also, the present paper aims to describe the studies analyzed uniformly and show their contributions in this field.
\end{abstract}

Keywords: Stakeholder Identification Process, software process, effective practices, software requirements, elicitation.

\section{Introduction}

Requirements Engineering (RE) as a discipline was developed when the quality of requirements was recognized as a key factor in preventing many of the causes leading to software failure. Measures taken at an early stage of a project can have great repercussions, and they are also more beneficial than those taken at later stages. The problem of the "software crisis" has, to a great degree, shifted to the area of requirements. Is there, then, some aspect of the requirements area that deserves particular attention? If so, this aspect should be taken into account at the initial stage of RE. Such is the case in requirements elicitation activities in which the problem to be solved is identified, and more importantly, the stakeholders must be identified. Relationships and ways of communicating between the development team and the customer are thereby established [1].

${ }^{1}$ The notion of a software crisis emerged at the end of the 1960s [24]. The term refers to difficulties in writing correct, intelligible and verifiable computer programs. The roots of the software crisis hinge around complexity, expectations, and change. Conflicting requirements have always been a hindrance to the software development process. 
However, identification of stakeholders as well as their needs and expectations are poorly done in software projects [2], probably because this process ${ }^{2}$ is mistakenly viewed as a self-evident task in which direct users, clients, and the development team, are the only stakeholders. It could also be due to the fact that the identification area can be obviated or substituted by opinions or knowledge obtained from other more accessible sources of information. In the short term, this would create less conflict of interests resulting from different points of view.

The findings in the problem statement are presented from two points of view: firstly, the impact of stakeholder identification on software requirements quality, and secondly, practices developed to carry out this task. So far, there has not been any SIP framework or uniform description. In view of this, the present paper we offers a uniform description of SIP as a first step towards developing a methodology that would cover all necessary aspects of stakeholder identification. Hence, at this stage, the present paper does not represent a technical solution.

\section{What Are the Consequences of Incorrect Stakeholder Identification on the Quality of Software Requirements?}

To answer the question raised, it is necessary to consider what Software Requirement Specification Quality (SRSQ) involves. The IEEE Standard 830 [3] gives a summary of the properties that should ideally be part of software requirement specification. Any identification process that mistakenly recognizes someone as a stakeholder will probably include requirements which do not correspond to any real need (a feature of "Correctness" of the standard). On the other hand, when the identification task fails to detect participants who are needed for the software project, requirement specifications are no longer "Complete" due to the omission of relevant requirements for project success, and this could give rise to inconsistent specifications. Failing to obtain these properties can create risks that could affect the project. Completeness, correctness and consistency in the RSQ can be ensured by applying proper elicitation techniques such as scenarios, use cases, etc. All of these, however, require a previous Stakeholder Identification Process (SIP).

The review of relevant initiatives from the field of Software Engineering (SE) and Information Systems (IS), referred to in Section 3 "Stakeholder identification as seen in previous studies of Software Engineering", confirms how all of these distinguish different types of stakeholders involved in software development, each type having different priorities and interests. To the same extent, all initiatives take stakeholder identification for granted and confine their task to indicating "who" they can be. They do not, however, clarify "how" the SIP process must be carried out to ensure getting correct stakeholders and thereby obtain accurate and complete requirements.

The implications of SIP on the quality of requirements are evident. This has not gone unnoticed, not even by those who have created standards or done studies that have been used as guidelines for software improvement processes. The comparison of

${ }^{2}$ The CMMi model [2] defines a process as a set of activities that can be recognized as implementations of practices. 
development studies shows that authors such as Wiegers [4] are more interested in identifying and applying effective practices ${ }^{3}$ than in obtaining complete solutions for project development.

There have been calls for different initiatives based on collections of data and experiences with proposals for effective practices that successfully meet users' needs.

The CMMi [2] does not explicitly mention any specific stakeholder identification practice. According to the CMMi, stakeholder needs are fundamental in determining customer requirements. These needs must be analyzed together with stakeholders' expectations, constraints, interfaces, operational concepts, and product concepts and be harmonized, refined, and transformed into a set of customer requirements. This goal includes specific practices such as collecting basic needs and also eliciting unarticulated needs. This involves the previous specification of stakeholders.

Does the lack of mention of a specific stakeholder identification practice imply a lack of precision in the description of the standard, or does it imply that the importance of stakeholder identification with respect to the problem is reduced? To answer this question, other proposals of effective practices will now be analyzed in detail.

\subsection{Effective Practices Recommended for Performing SIP and the Benefits of Proper Stakeholder Identification}

- Hoffman's [5] studies identify some RE effective practices. These practices measure the effectiveness of a software project and are organized according to the different factors which have an impact on their objective. Within all of these practices, the ones related to SIP (that is, Identify and consult all likely sources of requirements) are framed within the knowledge factor under the argument that stakeholders should meet demands in terms of experience and expertise for effective team work. It's a question, then, of a) carefully selecting team members who are skilled in the application domain, IT, and RE processes, b) always assigning experienced, capable project managers to RE, and c) consulting domain experts and stakeholders at an early stage of the process to increase and validate the team's knowledge.

- Wiegers [4] identifies a group of effective practices related to the elicitation task. One of these practices (namely, Identify user classes and their characteristics) emphasizes the need for stakeholder identification. There may, in fact, be many groups of customers who use the product, and these can be classified in terms of frequency of use of the product, use characteristics, levels of privileges, or levels of skills. Since each type of project (for example, commercial applications, integrated systems, web developments, etc.) requires different experts, proper selection of stakeholders is recommended. This selection involves previous assessment of stakeholders in terms of risk and cost, and also taking into account standard types of

\footnotetext{
${ }^{3}$ Effective practices are activities that people with recognized expertise in a particular area have identified from experience as making significant contributions to project success. Approximating effective practices enables us to create a kit with a variety of practices that can be applied to different problems [23].
} 
communication between users and developers. Communication, for example, in which developers can talk directly to potential users is more effective because it avoids loss of information generated by using intermediaries.

- Another approach to the specification of effective practices is the development of techniques and strategies which improve the RE processes. The REAIMS Project (8649) [6] set out to develop a maturity model for the RE similar to the SEI's CMM, that is, in terms of the scope to which an organization has defined the RE process based on effective practices. In this project, a set of basic guidelines is recommended for organizations that are at an initial stage of maturity. The guideline entitled "Identify and consult with the stakeholders of the system" recommends making a very specific list of stake-holders at an early stage of the RE process. It proposes a method of following the guidelines that ensures that only appropriate stakeholders are identified within each category of proposed stakeholder classifications. It is further suggested that an explicit list of stakeholders be drawn up and reasons given why the requirements will probably be important.

In summary, there is general agreement about the need to find effective practices relating to stakeholder identification in industry. So far, these practices have only defined different types of stakeholders on the basis of small groups of typical users and representatives of other people involved in the development of the project.

The benefits are evident: a proper selection of stakeholders improves the coverage of requirements, avoids overlapping of requirements in the user community, and allows for a more rational organization of requirements (Figure 1). In this way, people get involved more easily, and are less reluctant to implement the system and give information relating to requirements. However, effective practices, or standards such as CMMi, have the following limitations: they do not explain how to define the entire set of stakeholders. Furthermore, this process is not always self evident, and so the organization must be analyzed in order to encounter all possible stakeholders. Hence, the application of a stakeholder identification method sometimes becomes indispensable. This is developed in the following section.

\section{Stakeholder Identification in Previous Software Engineering Studies}

Software engineers need to identify, characterize, and handle all viewpoints of the different types of stakeholders [7]. Stakeholders, meaning all those involved in a project and have some interest in the software to be developed, may vary from one project to another. It is, therefore, always necessary to carry out an adaptation assessment of stakeholders' contributions and their vested interests in a project. In spite of the importance of identifying all the relevant parts (stakeholders) involved in a software project, the SIP area has received less attention than others in the SE.

Some of the main initiatives of SE recognize the existence of different types of stakeholders and who therefore need to be identified in each project. 


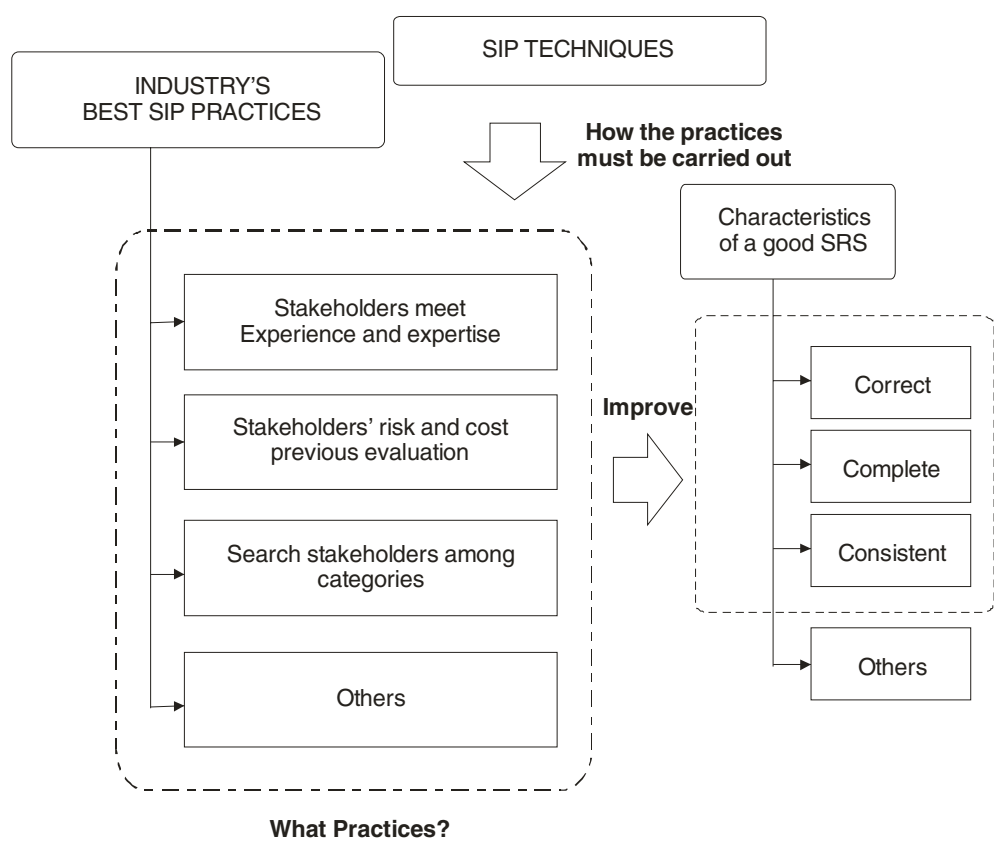

Fig. 1. Impact on the SIP effective practices on SRSQ

- The "Software Engineering Body Of Knowledge" (SWEBOK), emphasizes the description of the tasks related to each one of the proposed fields of knowledge, principally in what are called Requirements. For the SWEBOK, the process of requirements elicitation is a human activity in which stakeholders and relationships established between the development team and the client must be identified. [1]. RE is an interdisciplinary process in which all actors must become involved. Ignoring this can lead to the development of inappropriate systems. SWEBOK assigns the role of negotiator to the requirements engineer while other stakeholders are not taken into account.

- In an SEI Technical Report [8], stakeholders are identified at the requirements elicitation stage. They come from at least five communities involved in software development: clients/sponsors, users, developers, quality personnel, security personnel and the requirements analyst. The SEI's Capability Maturity Model Integration (CMMi- SW) [2] specifically proposes that stakeholders be selected from among customers, final users, developers, producers, test staff, suppliers, marketing staff, maintenance staff, and anyone who may affect or be affected by the software process and the final product. The CMMi defines two process areas related to RE: Requirements Management and Requirements Development. In each one of these areas, the different stakeholders involved are classified, and their different roles are defined on the basis of activities performed.

- In the standards developed by ISO and IEC, the ISO/IEC 12207 (software life cycle processes) provides a specific guideline to define the roles and responsibilities of some stakeholders in the life cycle of a software project, or product, or service. Some of the stakeholders mentioned are customers, quality personnel, software developers, etc. [9] 
- Ian Sommerville [10] situates the identification of stakeholders within the stages of obtaining and analyzing the software requirements. Among the identified stakeholders are the final users who will interact with the system, and also anyone inside the organization who will be affected by such a system. Stakeholders also include engineers who develop or support other related systems, for example, business managers, the IT specialist, workers' representatives, etc.

- Roger S Pressman argues that stakeholders must be identified in the beginning of the RE process because many different participants are involved at this stage. Pressman identifies the following stakeholders as being the most common: business managers, brand managers, marketing staff, external and internal customers, consultants, product engineers, software engineers, sup-port and maintenance engineers, etc. [11].

- In the Rational Unified Process (RUP) within the SE process, stakeholder identification is carried out at the management requirements processing stage. The most obvious stakeholders in a software project are: the final user, the software developer, the purchaser, the project director, and anyone strongly interested in the project or those who need the project to solve their needs [12].

The aforementioned studies confirm the variety of existing stakeholders involved in software development, each having different priorities and interests. All of these studies take SIP for granted and don't go beyond indicating "who" the stakeholders may be. They do not mention "how" the process must be carried out to properly identify stakeholders as a prerequisite to obtaining exact and complete requirements.

\section{What Is the Scope of Approximations in SIP Studies?}

So far, we have explained how each software project may have different types of stakeholders, and how selecting them appropriately has a strong impact on software requirements quality, and consequently, on the success of the software project itself. The studies reviewed could give the impression that many attempts have been made to define and give detailed explanations of how the SIP is done. This, however, is not the case. Currently, stakeholder identification methods are few and since the process is described by each author the SIP lacks a common framework of study and a uniform description. The studies described so far in this paper seem to only partially solve the issue of stakeholder identification.

Based on RE literature relating to stakeholder identification processes, we propose to group them into three categories. Section 4.1 gives the first category of studies which limit themselves to only proposing a list of possible stakeholders. Section 4.2 presents the second category of studies which not only indicate who the stakeholders can be, but also studies their interactions. The third category, in section 4.3, deals with studies that include an assessment of stakeholders.

\subsection{Studies That Exclusively Characterize Stakeholders}

These studies provide a list of potential stakeholders from which it is possible to determine which ones are really relevant and how each one may be contacted. These, however, are incomplete because they only provide a helpful guide to establish a final list of stakeholders. What must not be overlooked is that stakeholders will normally 
have to contribute their effort, time and/or money, and they must therefore know what benefits can be gained in return. Potential stakeholders must therefore be characterized by gathering relevant information about them. This information may also be useful for evaluating a set of identified stakeholders, and for obtaining new and more appropriate configurations. Some examples are:

- Lauesen [13] only summarizes this information from responses to just three questions: what goals do they identify for the system? Why would they like to contribute? Or, what risks and costs do they envisage?

- Robertson's VOLERE template [14] is used during the whole requirements process for other purposes such as assessing quality and specifying business requirements. The template is a tool that helps to discover the difference between a stakeholder's wish and a real need, and helps to establish the range of a system. This fundamental aspect of the process ensures that all stakeholders know what is and what is not within their domain. However, it can also be used to get a general idea about the stakeholders who are participating in the development of a system. This method only differentiates between the client and the buyer, and thereby ignores other stakeholders. Other stakeholders may be found in the following categories: users, sponsors, test staff, business analysts, technology experts, system designers, marketing experts, legal experts, IT specialists, usability experts, representatives of external associations, etc. Each of the stakeholders taken into account is identified in terms of who he/she is and what role he/she will play. They are selected from a list of stakeholders initially proposed and on the basis of knowledge which may be necessary for the project.

In general, these studies cannot be regarded as identification of stakeholders because they only provide information that makes it easy to identify them. They do not ensure that all the necessary stakeholders are detected.

\subsection{Studies Focusing on Interaction Between Stakeholders}

Once we have an idea of who the main stakeholders are, the basic interactions between these actors should be identified. This enables stakeholders to clarify which part of the problem falls within each one's scope. The following range of studies deal with this aspect.

- Smith [15] proposes a context diagram to enable stakeholders to see what is happening in the system. This starts with a brainstorming activity in which all stakeholders must be taken into account. At the center of the diagram, an oval represents the project itself and a horizontal line divides the stakeholders. The upper section contains stakeholders who belong to the organization such as clients, functional departments, team members, etc. The lower part contains external staff such as advertising agencies (press, radio and television), competitors, citizens, government organizations or representatives.

- Coakes and Elliman [16] develop a method to identify stakeholders and their different viewpoints in a computer information system using a legacy system. The authors use a web that has a system of classification and stakeholders are grouped by using a holistic view of the situation. This facilitates an understanding and identification of agreements between stakeholder interests. The web not only 
identifies stakeholders, it is also used to analyze relationships between activities which must be performed by the stakeholders and the members of the new system, with a view to prioritizing the proposed requirements. The system must be defined in terms of different boundaries: automation, technical, and total or human boundaries. Each boundary represents a wider view of the system and its impact. These limits are very generic and provide a general guideline as to who may be found within each limit. Stakeholder identification is a process that explores the web plane looking for interested parties. If stakeholders are identified, they are added to the web and thereby groups of stakeholders, needs and interests can be recognized.

- Sharp, Galal and Finkelstein [17] propose an approach to discover all stakeholders in the development of a specific software project. This identification is carried out by establishing a set of "baseline" stakeholders. From these, the "supplier" stakeholder (who provides information or supporting tasks to the baseline stakeholders) can be recognized, and also the "client" stakeholder (who examines products). Other stakeholders called "satellites" inter-act in various ways with the baseline stakeholders. "Interaction" may involve communicating, reading a set of rules or guidelines, searching for information, etc. Roles are assigned on the basis of an analysis of the interactions that can exist between different stakeholders and between the stakeholder and the system. Only the baseline stakeholder, however, is identified. The roles that they can perform are: users, developers, legislators, and decision makers.

- Preiss and Wegmann [18] adopt some fundamental principles of Systems Science to provide a generic, scientifically recognized basis that can aid stakeholder identification and classification. This method provides a framework that theoretically guarantees the identification of a complete set of relevant, abstract concepts and also all the stakeholders. The authors propose a generic stakeholder classification that is based on three principles: two systems, two viewpoints and two domains of enquiry. The software development life-cycle is divided into two stages: creation and operation, or in more concrete terms, the system creation stage (which includes conception, design and implementation), and the system operation stage (which includes system application in a real environment). Using this generic scheme, external and internal stakeholders can be identified.

\subsection{Studies That Include Assessment of Stakeholders}

- Mitchell, Agle and Wood [19] developed a theory of stakeholder identification based on the premise that identification is realized through an analysis of 'what' and 'who' affects the organization and also taking their salience into account. This identification is performed by analyzing stakeholders' interests in the project and considering three dynamic qualities, a) the power that the stakeholder has to propose requirements within the organization, b) legitimacy of the actions which a person performs within a certain social sys-tem constructed with the definition of norms, and c) urgency - meaning the degree of attention that a stakeholder claims from the project manager. Afterwards, possible stakeholders are grouped into three classes based on the degree of requirements priority: (a) latent or low salience, (b) expectant or moderate salience, and (c) definitive or high salience. Any stakeholder can become a definitive stakeholder by acquiring the missing attributes. Finally, the project 
manager determines who will be the 'feasible' stakeholder to include in the project, by analyzing a variety of existing stakeholder classes.

- The aim of the Method Engineering with Stakeholder Input and Collaboration, MEWSIC [20] (Young, McDonald, Edwards and Thompson), was to provide software developers with a practical tool to identify stakeholders. The method groups all the people involved in a project depending on the priorities of their interests. In stakeholder analysis, the MEWSIC approach suggests the identification of people related to the project and an assessment of their relevance to the project being developed to determine if he/she should or should not be taken into account. Appropriate stakeholders are those who have not only relevant knowledge and skills but also have suitable attitudes towards the process; some stakeholders, for example, are not suitable for negotiating. For this reason, this method proposes the use of personality tests to complement stakeholder analysis and make it possible to achieve an adequate fit between the stakeholder, the system context, and the characteristics of the development project. Furthermore, the method introduces group dynamics to observe stakeholder behavior within groups and how this affects a member's performance.

- McManus' approach analyzes the guide proposed by the World Bank (WB) model in 1996 for stakeholder identification [21]. The goal of this stakeholder analysis is to identify stakeholder categories using a WB questionnaire, to develop a strategic view of the situation and the relationship between different stakeholders and identified objectives, and to explain stakeholder interests and roles. The questionnaire results should provide information about stakeholders and their interests, the relationship between them, their motivations, and their ability to influence outcomes. The WB model proposes four groups of stakeholders. Primary stakeholders include those who, because of power, authority, responsibilities or claims over the resources, are primordial for any project initiative. Secondary stakeholders are those who have an indirect interest in the outcome. External stakeholders come from outside the project and expect something from the project team. And finally, the Extended stakeholders may often be of help to primary and secondary stakeholders to reach a unified vision and develop feasible plans of action. The roles of the four groups are established by means of the following activities: collecting and analyzing information, defining priorities and establishing goals, assessing available resources, project planning, designing strategies to implement these programs and dividing responsibilities among participants who manage a project, monitor progress, and evaluate results and impacts. However, since the identified stakeholders may not have enough capacity to participate in the project, each one's strengths and weaknesses is identified in order to form collaboration groups.

\section{Conclusions}

During software requirements elicitation we decide what exactly is to be produced. At this stage, the appropriate identification of stakeholders is vitally important as a means of understanding the environment in which the software project will be developed and operated, and also to identify which stakeholders will participate in the project. This is a key aspect in the process of obtaining the expected quality 
requirements specifications, in the sense that they must be appropriate, complete, and free of contradictions. This means that all stakeholders need to have appropriate knowledge and none stakeholder can be omitted. Good interaction is vital during the requirements-gathering process, and also between all stakeholders and the system to avoid conflicts and problems of communication arising from different points of view.

The state-of-the-art of SIP referred to in the present paper shows different interpretations of the scope of this process. All of the software initiatives referred to assume a way of contributing to the improvement of the software process by implementing a set of good industry practices for RE that have been identified, acknowledged, and disseminated, however they have not explained how to carry out the SIP. Some initiatives provide numerous examples of who can be stakeholders by establishing generic categories into which they may be grouped.

Other studies analyzed are more ambitious. However, the studies mentioned in this paper are not standardized and consequently the SIP is not standardized either. Not all of them, however, cover the same aspects and are not applicable to the same situations. This makes it difficult to select a correct stakeholder identification method be-cause some methods only characterize stakeholders but without assigning a stakeholder's role in a specific project (Lauesen [13] and Robertson's VOLERE template [14]); others like Smith [15], Coakes and Elliman [16], Sharp, Galal and Finkelstein [17] and Preiss and Wegmann [18] analyze stakeholder interaction but they do not cover human aspects of stakeholder identification (e.g. personality tests, human behavior). Only a few methods include stakeholder assessment (Mitchell, Agle and Wood [19], MEWSIC [20] and McManus [21]). Furthermore, not all the studies analyzed take into account aspects such as when and how we know that the stakeholders identified are sufficient for the project, and how all the information collected will be documented.

The SIP must take into account the impact of personality types and the roles they may play. Stakeholders are assessed in terms of their characteristics, the knowledge needed, their influence on a project, and the relationships between stakeholders. SIP must also develop schemes to characterize and evaluate appropriate relationships between all stakeholders. For example, labels such as "one person is in charge of", "this person is an assistant to", "he/she is crucial for", "he/she provides the information for" could be used.

Up to now, the SIP continues receiving very little attention from the different existing initiatives in software development (for example, CMMi, SWEBOK, the IEEE Standard 830), despite the fact that success in software products depends to a great deal on proper stakeholder identification in requirements specification. Therefore, on the basis of this literature survey, as further research we propose:

- The validation of all empirical studies analyzed in a specific project; this will enable us to determine its effectiveness.

- The development of a guide which would recommend the use of a specific method of stakeholder identification based on the particular characteristics of the project to be developed.

- Develop a new methodology to adequately perform the SIP by covering the shortcomings found in the methods analyzed in this paper. 


\section{References}

1. International of Electrical and Electronics and Electronics Engineers. Guide to the Software Engineering Body of Knowledge. New York (2004) www.swebok.org

2. Software Engineering Institute, Capability Maturity Model ${ }^{\circledR}$ Integration (CMMI®). Version 1.1, CMMI-SE/SW/IPPD/SS V1.1. Technical Report CMU/SEI-2002-TR-011 or ESC-TR-2002-011. Software Engineering Institute. Pittsburg, PA (March 2002)

3. IEEE. Guide to Software Requirements Specification, IEEE Std. 830-1998. IEEE Press. Piscataway, NJ (1998)

4. Wiegers, K.E.: Software Requirements. Microsoft Press. ISBN 0-7356-0631-5 (1999)

5. Hofmann, H.F., Lehner, F.: Requirements Engineering as a Success Factor in Software Projects. IEEE Software, July/August 2001 (2001)

6. Sommerville, P.S.: Requirements Engineering. A Good Practice Guide. J. Wiley \& Sons Ltd. ISBN 0471974447 (1997)

7. Kotonya, G., Sommerville, I.: Requirements Engineering: process and techniques. John Wiley and Sons, New York (2000)

8. Software Institute Requirements Engineering Project. Issues in Requirements Elicitation. Technical Report CMU/SEI-92-TR-012 or ESC-TR-92-012. Software Engineering Institute, Pittsburg, PA (September 1992)

9. Lawson, H.W.: Defining Stakeholder Relationships. IEEE Computer Society, Washington (1999)

10. Sommerville, I.: Ingeniería de Software. In: 6a Edición, Addison-Wesley, London (2002)

11. Pressman Roger, S.: Software Engineering: A Practicioner's Approach, 6th edn. Mc Graw Hill, New York (2005)

12. Kruchten, P. (ed.): The Rational Unified Process an Introduction, 3rd edn. Addison Wesley, London (2003)

13. Lauesen, S.: Software Requirements: Styles and Techniques. In: Pearson Education, ISBN 020174570 4, Addison-Wesley, London (2002)

14. Robertson, S., James, R.: Mastering the requirements process. Addison-Wesley, London (1999)

15. Smith, L.W.: Project clarity through stakeholders analysis. The Journal of Defense Software Engineering. Issue (December 2000) http://stsc.hill.af.mil/crosstalk/2000/12/smith.html

16. Elayne, C., Tony, E.: The role of the stakeholder in managing change. Communications of AIS, vol. 2, Article 4 (July 1999)

17. Sharp, H., Galal, G.H., Finkelstein, A.: Stakeholder Identification in the Requirements Engineering Process. In: Database \& Expert System Applications, IEEE Press, New York (1999)

18. Preiss, O., Wegmann A.: Stakeholder Discovery and Classification Based on Systems Science Principles. Quality Software, 2001. IEEE Proceedings. Second Asia-Pacific Conference On, December 10-11, 2001. pp. 194-198 (2001)

19. Mitchell, R.K., Agle, B.R., Wood, D.J.: Toward a Theory of Stake-holder Identification and Salience: Defining the Principle of Whom and What Really Counts. Academy of Management Review. vol. 22(4) (1997)

20. Young, M., McDonald, S., Edwards, H.M., Thompson, J.B.: Quality \& People in the Development of Situationally Specific Methods. Quality Software. 2001, Proceedings IEEE. Second Asia-Pacific Conference On December 10-11, 2001, pp. 199-203. (2001)

21. McManus, J.A: Stakeholder Perspective within Software Engineering Projects. In: Engineering Management Conference on Proceedings, IEEE International, October 18-21, 2004, vol. 2. pp. 880-884 ( 2004) 